\title{
Modulation of Calcium-activated Potassium Channels from Rat Brain by Protein Kinase $A$ and Phosphatase $2 \mathrm{~A}$
}

\author{
Peter H. Reinhart, ${ }^{1}$ Sungkwon Chung, ${ }^{1}$ Bruce L. Martin,, ${ }^{2}$ David L. Brautigan, ${ }^{2}$ and Irwin B. Levitan ${ }^{1}$ \\ ${ }^{1}$ Graduate Department of Biochemistry and Center for Complex Systems, Brandeis University, Waltham, Massachusetts \\ 02254 and 'Division of Biology and Medicine, Brown University, Providence, Rhode Island 02912
}

By incorporating plasma membrane vesicles into planar lipid bilayers, we previously characterized a family of four types of $\mathrm{Ca}^{2+}$-activated $\mathrm{K}^{+}$channels from rat brain (Reinhart et al., 1989). Two of these are "large-conductance" or "maxi"'-K+ channels, which differ in their gating kinetics and toxin sensitivity and are henceforth referred to as "type 1" and "type 2" channels. Here we show that the gating of these two channel types can be modulated by phosphorylation and dephosphorylation. The effects of CAMP-dependent protein kinase catalytic subunit (PK-A) on type 1 maxi-K ${ }^{+}$channels are complex in that, while half of these channels are upregulated by the kinase, about one out of seven channels is downregulated. Thus, there may be several distinct channels within the type 1 category. Type 2 maxi-K+ channels are consistently downregulated by PK-A. The effects of PK-A on both channel types are reversed by the catalytic subunit of protein phosphatase 2A (PP-2A), but not by protein phosphatase 1 (PP-1). Furthermore, some of the type 1 maxi-K+ channels can be modulated by PP-2A, even without any prior PK-A treatment, indicating they are in a phosphorylated state when they are incorporated into the bilayer. The results demonstrate that (1) type 1 and type 2 maxi-K+ channels are substrates for PK-A; (2) phosphorylation can shift the open probability of channels in either direction, by a mechanism involving multiple phosphorylation sites; (3) phosphorylation alters the $\mathrm{Ca}^{2+} /$ voltage sensitivity of these channels; and (4) dephosphorylation of type 1 and type 2 channels is catalyzed by specific phosphatases.

$\mathrm{Ca}^{2+}$-activated $\mathrm{K}^{+}$channels can be divided into a number of subclasses based on properties such as single-channel conductance, voltage dependence, the sensitivity to pharmacological agents such as tetraethylammonium (TEA), apamin, and scorpion toxins, and inactivation kinetics (Hille, 1984; Petersen and Maruyama, 1984; Elkins et al., 1986; Moczydlowski et al., 1988; Rudy, 1988; Castle et al., 1989; Reinhart et al., 1989). Some cells possess more than one type of $\mathrm{Ca}^{2+}$-activated $\mathrm{K}^{+}$channel, each type apparently playing a different role in the overall regulation of the cell's electrical activity (Romey and Lazdunski,

\footnotetext{
Received Oct. 9, 1990; revised Dec. 31, 1990; accepted Jan. 4, 1991.

We are grateful to Chris Miller for his interest and advice throughout the course of this work and to Terry Egan and Paul Katz for helpful comments on an earlier version of the manuscript. This work was supported by NIH Grants NS17910 to I.B.L., GM31768 to C. Miller, and DK31374 to D.L.B.

Correspondence should be addressed to Dr. Irwin B. Levitan, Graduate Department of Biochemistry and Center for Complex Systems, Brandeis University, 415 South Street, Waltham, MA 02254.

Copyright (C) 1991 Society for Neuroscience $0270-6474 / 91 / 111627-09 \$ 03.00 / 0$
}

1984; Pennefather et al., 1985; Blatz and Magleby, 1986). These channels are intriguing because they provide a link between second-messenger systems, such as the inositol triphosphate $\left(\mathrm{IP}_{3}\right)$ induced release of intracellular $\mathrm{Ca}^{2+}$, and the membrane potential. Some members of this channel subgroup are also targets for other intracellular modulators such as protein kinase $\mathrm{C}$ (Baraban et al., 1985; Doerner et al., 1988; Kaczmarek, 1988; Shearman et al., 1989) and protein kinase A (PK-A; De Peyer et al., 1982; Ewald et al., 1985; Lechleiter et al., 1988; Kume et al., 1989; Sikdar et al., 1989).

Using reconstitution techniques, we have described two distinct types of maxi-K ${ }^{+}$channels in brain plasma membrane preparations (Reinhart et al., 1989). These two channel types share a number of properties such as the unitary conductance, voltage dependence, and TEA sensitivity. The main differences are that type 1 or "fast-gating" channels are blocked by charybdotoxin (CTX), while type 2 or "slow-gating" channels show different gating kinetics and are not blocked by nanomolar concentrations of CTX. The ability to incorporate these two channel types from brain plasma membrane vesicles into planar lipid bilayers opens the possibility to study the modulation of these channels in a defined environment, devoid of interactions with any but tightly associated intracellular components. In the present study, we show that protein phosphorylation by PK-A can modulate the gating kinetics of type 1 and type 2 maxi-K $\mathrm{K}^{+}$ channels. The direction of modulation is dependent on the individual channel type. These effects of PK-A can be reversed by protein phosphatase $2 \mathrm{~A}$ (PP-2A), but not by protein phosphatase 1 (PP-1). The effect of such phosphorylation and dephosphorylation cycles may be to adjust precisely the $\mathrm{Ca}^{2+}$ voltage sensitivity of target ion channels.

Some of these data have been previously presented in abstract form (Reinhart and Levitan, 1987).

\section{Materials and Methods}

Vesicle preparation. Rat brain plasma membrane vesicles were prepared as described previously (Reinhart et al., 1989). Briefly, brains were rapidly excised and immediately homogenized in an ice-cold isotonic sucrose buffer $[0.25 \mathrm{~m}$ sucrose, $2.5 \mathrm{~mm} \mathrm{KCl}, 0.1 \mathrm{~mm}$ EGTA, $0.1 \mathrm{~mm}$ dithiothreitol, and $20 \mathrm{~mm}$ HEPES ( $\mathrm{pH}, 7.2)]$. The homogenate was centrifuged at $800 \times g$ for $3 \mathrm{~min}$, the pellet was washed once, and the supernatants were centrifuged at $30,000 \times g$ for $10 \mathrm{~min}$. The pellet was resuspended in a hypotonic "lysis buffer" and allowed to stand on ice for $20 \mathrm{~min}$. The membranes were then rehomogenized, pelleted, and layered under a discontinuous step gradient of Percoll $(25 \%, 18 \%, 10 \%$, and $0 \%, v / v)$. The gradients were centrifuged at $45,000 \times g$ for $2 \mathrm{~min}$, and the membrane fractions banding at the $0-10 \%$ interface were collected. The Percoll was removed by centrifugation, and the membranes were frozen at $-70^{\circ} \mathrm{C}$.

Planar bilayers and membrane vesicle fusion. Single $\mathrm{Ca}^{2+}$-activated 
$\mathrm{K}^{+}$channels were inserted into planar lipid bilayers (Mueller and Rudin, 1969) formed from a mixture of 1-palmitoyl, 2-oleoyl-phosphatidylethanolamine (PE) and 1-palmitoyl, 2-oleoyl-phosphatidylserine (PS; $3: 1$ ) in $n$-decane, as described previously (Reinhart et al., 1989). Membranes were added to the cytoplasmic solution consisting of $150 \mathrm{~mm}$ $\mathrm{KCl}, 1.05 \mathrm{mM} \mathrm{CaCl}_{2}, 1 \mathrm{~mm}$ EGTA, $1 \mathrm{~mm} \mathrm{MgCl}, 7 \mathrm{~mm} \mathrm{KOH}$, and 10 mM IIEPES (plI, 7.2). The extracellular solution consisted of $0.1 \mathrm{~mm}$ EGTA, $7 \mathrm{~mm} \mathrm{KOH}$, and $10 \mathrm{~mm}$ HEPES (pH, 7.2). For experiments involving the addition of PK-A, $500 \mu \mathrm{M} \mathrm{Mg-ATP}$ was added to this solution. For some experiments, the $\mathrm{Ca}^{2+}$ concentration was adjusted by adding more EGTA or more $\mathrm{CaCl}_{2}$. $\mathrm{Ca}^{2+}$-activated $\mathrm{K}^{+}$channels inserted predominantly in one orientation, that is, with the $\mathrm{Ca}^{2+}$-sensing side facing the cytoplasmic solution. After the incorporation of a channel, further fusion was suppressed by adding $50 \mathrm{mM} \mathrm{KCl}$ to the extracellular side, thereby reducing the transmembrane osmotic gradient. The electrical conductance of the bilayer was measured under voltageclamp conditions at room temperature. Voltages are expressed in cellular convention, that is, the voltage of the cytoplasmic side with respect to the extracellular side. Solution changes were made by perfusing either the cytoplasmic or the extracellular chambers.

Electrical recordings and analysis. Single-channel currents were amplified using a $10-\mathrm{G} \Omega$ feedback resistor, filtered at $1-4 \mathrm{kHz}$ using an 8 -pole Bessel filter, and recorded on a $\beta$-videocassette recorder after 16 bit digitization at $44 \mathrm{kHz}$ with a pulse code modulator (Sony PCM-701 ES). Data were analyzed off line using an MS-DOS-compatible computer (Everex 386, $20 \mathrm{MHz}$ ) equipped with a Tecmar Labmaster A/D board coupled to an Indec IBX Instrumentation Interface, and utilizing an Indec BASIC-Fastlab software library. Using operator-assisted computer programs, single-channel currents were sampled at either 4 or 8 $\mathrm{kHz}$ (unless otherwise indicated) and searched for transitions away from baseline. The threshold for detecting opening and closing transitions was set to $50 \%$ of the open-level current for each individual event. In this way, current levels corresponding to each closed/open transition were reduced to three values corresponding to the closed time, open time, and amplitude. Such reduced data lists were then further transformed to yield the open probability, mean amplitude, closed-time distributions, and open-time distributions. All experiments were repeated at least four times.

Kinase and phosphatase preparations. The catalytic subunit of the cAMP-dependent protein kinase was prepared from bovine heart essentially as described previously (Demaille et al., 1977; Peters et al., 1977). The final purification was achieved by HPLC using a Du Pont GF-250 gel filtration column. Catalytic subunits of protein phosphatases 1 and $2 \mathrm{~A}$ were prepared by a modification of the procedure of Brautigan et al. (1985) as described by Pallis et al. (1990).

Materials. All lipids were from Avanti Polar Lipids (Birmingham, AL). Percoll was from Pharmacia (Boston, MA). Decane was from Aldrich (Milwaukee, WI)

\section{Results}

Rat brain $\mathrm{Ca}^{2+}$-activated $\mathrm{K}^{+}$channels were incorporated into planar lipid bilayers (PE/PS, 3:1) and clamped at $+10 \mathrm{mV}$ using asymmetric ionic conditions ( 150 vs. $50 \mathrm{mM} \mathrm{KCl}$, cytoplasmic vs. extracellular). Previous experiments have shown that under such conditions two different types of maxi- $\mathrm{K}^{+}$channels can be incorporated (Reinhart et al., 1989). The channels can be differentiated on the basis of a number of criteria. Type 1 channels are characterized by shorter mean open times and shorter mean closed times; that is, they appear to be fast-gating channels and are blocked by nanomolar concentrations of CTX. Type 2 channels are slow gating, less sensitive to $\mathrm{Ca}^{2+}$ by a factor of 2-5, and are not blocked by CTX. Hence, we can unequivocally assign brain maxi- $\mathrm{K}^{+}$channels as being either type 1 or type 2 .

Shown in Figure 1 is a single-channel record of a type 1 maxi$\mathrm{K}^{+}$channel (openings appear as upward deflections). Following addition of $400 \mathrm{nM}$ PK-A, there was an upregulation of type 1 channels in 22 of 43 experiments, resulting in the channel being open most of the time (Fig. 1, center trace). Addition of $30 \mathrm{~nm}$ catalytic subunit of PP-2A resulted in the reversal of the PKA-induced upregulation of this maxi-K $\mathrm{K}^{+}$channel (Fig. 1, bottom trace) in five of six experiments. Addition of comparable levels of PP-1 instead of PP-2A was without effect (four experiments; data not shown). We often observed that, after PK-A upregulation, the addition of PP-2A lowered the open probability of channels to below control levels (cf. Fig. 1, top and bottom (races). Presumably, the channel is incorporated in a partially phosphorylated form prior to PK-A addition, and these endogenous phosphates can be removed by the addition of PP-2A.

In 15 of 43 experiments with type 1 channels, PK-A had no significant effect on channel gating. To investigate whether this resulted from the incorporation of already highly phosphorylated type 1 channels, we examined the effects of adding PP-2A in the absence of any added PK-A. The data in Figure 2 show that adding PP-2A to type 1 channels prior to the addition of any PK-A caused a pronounced decrease in the open probability of the channel (four out of six experiments). To confirm that PP-2A addition after PK-A treatment (Fig. 1) does indeed reverse the actions of the kinase and is not removing endogenous phosphate groups present on the channel prior to PK-A administration, we first treated a type 1 channel with PP-2A and then perfused the cytoplasmic side of the channel extensively. After this treatment, we then added PK-A to activate the channel. Under these conditions, addition of PP-2A is still able to reverse the effect of PK-A (data not shown). In contrast to these results with type 1 channels, PP-2A added to type 2 channels prior to the addition of PK-A is without effect (four out of four experiments). Heat-inactivated PP-2A is without effect on either type 1 or type 2 channels. In all experiments, there was a time lag ranging between $30 \mathrm{sec}$ and $3 \mathrm{~min}$ from the addition of either PK-A or PP-2A to the change in the activity of the channel, consistent with an enzymatic reaction taking place.

Initially we observed that PK-A significantly decreased the open probability of type 1 channels in one out of eight experiments. Increasing the number of experiments, we found this response in 6 of 43 experiments. Therefore, it seems that a subgroup of type 1 (CTX-sensitive) channels is downregulated by PK-A. This subgroup appears to represent a channel type that cannot be distinguished from the majority of type 1 channels by the criteria of gating kinetics and toxin sensitivity.

In contrast to the upregulation observed with most type 1 channels, the addition of PK-A to type 2 maxi-K ${ }^{+}$channels resulted in a decrease in the open probability of these channels in seven out of eight experiments (Fig. 3, center trace). Again, addition of PP-2A reversed these effects on type 2 channels (four out of four experiments; Fig. 3, bottom trace). Hence, the effects of PK-A and PP-2A on this channel type are opposite to that on type 1 maxi-K $\mathrm{K}^{+}$channels. Note that the open probability of the channel after kinase and phosphatase treatment is about the same as the open probability of the untreated channel (Fig. 3, top and bottom traces).

The data in Figures 4 and 5 show the time dependence of the PK-A and PP-2A effects on single-channel open probabilities. For these experiments, single-channel fluctuations were measured in 60 -sec sweeps, and the channel open probability for that sweep was calculated. The addition of PK-A to type 1 channels (Fig. $4 A$ ) results in a steep rise in the open probability of the channel, peaking within 5 min of administration. In these experiments, the open probability of the channel after PK-A administration is not constant, but oscillates between the control value and a much higher open-probability value $\left(p_{o}\right)$. The open probability of type 1 channels is reduced within minutes of PP$2 \mathrm{~A}$ addition. Note that the $p_{o}$ after PP-2A addition is lower than 


\section{Type-1 Channel}

\section{Control}

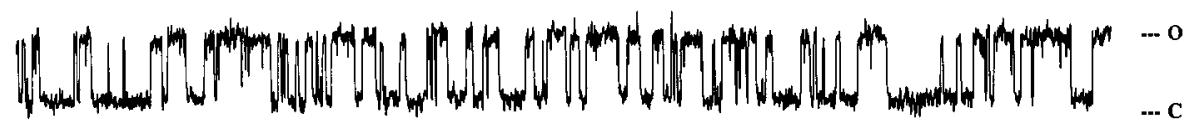

PK-A

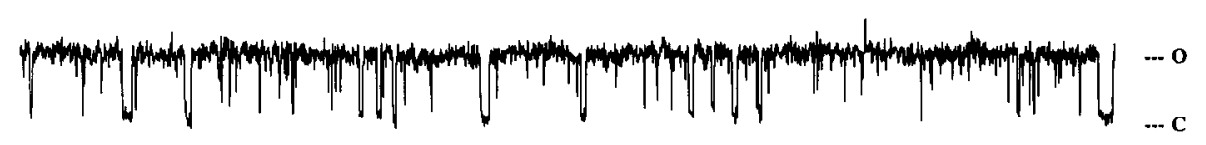

PK-A + PP-2A

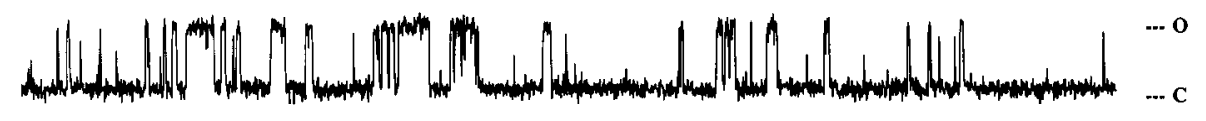

$$
\frac{}{200 \mathrm{~ms}} 5 \mathrm{pA}
$$

Figure 1. Upregulation of type $1 \mathrm{Ca}^{2+}$ activated $\mathrm{K}^{+}$channels by cAMP-dependent protein kinase and reversal of this effect by PP-2A. Single-channel current records were obtained by incorporating plasma membrane vesicles from rat brain into PE/PS (3:1) planar bilayers. To facilitate detection and characterization of ion channels, asymmetric salt conditions were used (150 $\mathrm{mM} \mathrm{KCl}$ on the cytoplasmic side, 50 $\mathrm{mM} \mathrm{KCl}$ on the extracellular side). The free $\mathrm{Ca}^{2+}$ concentration was adjusted to approximately $0.5 \mu \mathrm{M}$ using either $\mathrm{CaCl}_{2}$ or EGTA, and the voltage across the bilayer was clamped at $+10 \mathrm{mV}$. For all traces, channel openings $(O)$ are shown as upward deflections from the closed-level current $(C)$. The top trace shows a 2.25-sec control sweep just prior to the addition of PK-A; the center trace, a 2.25 -sec sweep $3 \mathrm{~min}$ after the addition of $400 \mathrm{nM}$ PK-A; and the bottom trace, a 2.25 -scc swccp obtaincd 3 min after the addition of $30 \mathrm{nM}$ of PP$2 \mathrm{~A}$ and while still in the presence of PKA. Current fluctuations were filtered at $2 \mathrm{kHz}$ and digitized at $4 \mathrm{kHz}$. the control value ( 0.1 vs. 0.3 ; Fig. $4 A ; 0-20$ min vs. $45-60 \mathrm{~min}$ ). Furthermore, after PP-2A treatment, there was no more obvious oscillation in the open probability. The addition of the catalytic subunit of PP-1 instead of PP-2A never caused significant shifts in the open probability, whether added alone or after PK-A treatment. In the absence of PK-A or PP-2A, we never observed rapid, large changes in the open probability of single ion channels (Fig. 4B).

The effects of PK-A and PP-2A treatment on type 2 channels are shown in Figure $5 A$. After addition of PK-A, there is a steep decrease in the single-channel open probability, reaching a new steady state within $5 \mathrm{~min}$. The new open probability tends to be constant, without the large oscillations observed for type 1 channels. Addition of PP-2A produces a sharp increase in $p_{o}$, reversing the effects of $\mathrm{PK}-\mathrm{A}$ by restoring the $p_{o}$ to initial values over a 5-min period. Thus, the effects of PK-A and PP-2A appear to be mirror images on type 2 channels. Even though spontaneous open-probability shifts for type 2 channels are typically larger than those observed for type 1 channels, we never observed sudden large open-probability shifts in the absence of

\section{Type-1 Channel}

\section{Control}

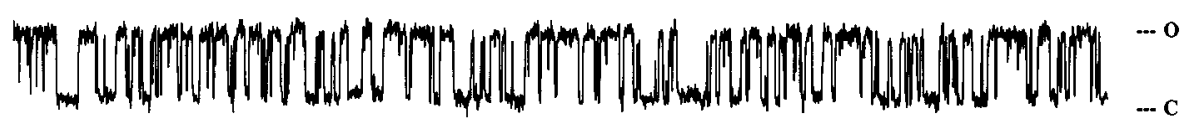

PP-2A

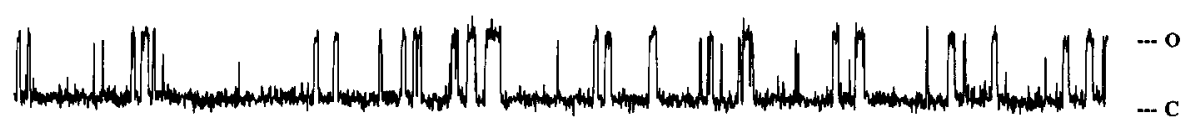

Figure 2. PP-2A alone can inactivate type $1 \mathrm{Ca}^{2+}$-activated $\mathrm{K}^{+}$channels. Single type $1 \mathrm{Ca}^{2+}$-activated $\mathrm{K}^{+}$channels were incorporated into lipid bilayers and clamped at $+10 \mathrm{mV}$ as described in Figure 1. The top trace shows a 2.25sec sweep of a control channel, prior to any kinase or phosphatase treatment; the bottom trace, a $2.25-\mathrm{sec}$ sweep 3 min after the addition of $30 \mathrm{nM}$ PP-2A. 


\section{Type-2 Channel}

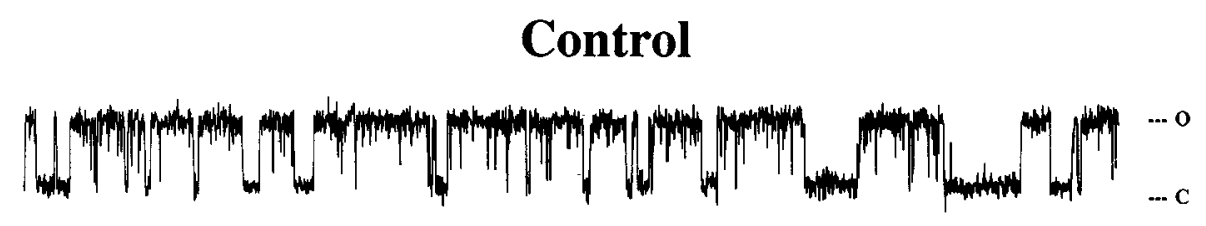

Figure 3. Downregulation of type 2 $\mathrm{Ca}^{2+}$-activated $\mathrm{K}^{+}$channels by cAMPdependent protein kinase and reversal of this effect by PP-2A. Single-channel currents were obtained as described in Figurc 1. The top trace shows a 2.25 sec control sweep of a channel clamped at $+10 \mathrm{mV}$; the center trace, a $2.25-\mathrm{sec}$ sweep $3 \mathrm{~min}$ after the addition of 400 nM PK-A; and the bottom trace, a $2.25-$ sec sweep $3 \mathrm{~min}$ after the addition of $30 \mathrm{nM}$ PP-2A and while still in the presence of PK-A.
PK-A
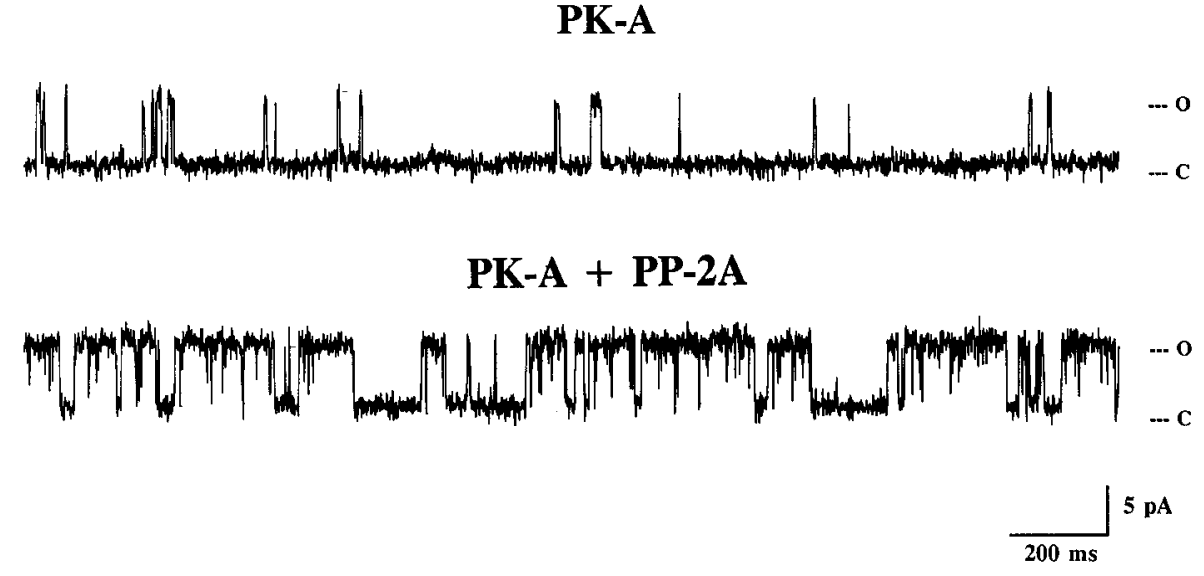

added PK-A or PP-2A (Fig. $5 B$ ). We never observed any effects of heat-inactivated PK-A $(n=3)$ or of PK-A added in the absence of $\operatorname{ATP}(n=4)$.

An analysis of the open-time and closed-time distributions (Table 1) revealed that both type 1 and type 2 channels can be described by three closed states and two open states. The fit for the open-time distribution for type 1 channels was only slightly improved by fitting to a sum of two rather than a single exponential term. Table 1 shows that the effect of PK-A on type 1 channel gating is to shorten closed times and increase open times, though the change in closed times is predominant. There appears to be little cffect on the fastest "flickery" closed state, but this state is not clearly resolved in our experiments. It can also be seen that PP-2A modifies the same kinetic parameters as PK-A but in the opposite direction. The effect of PK-A on type 2 channels is also to alter both the open-time and the closedtime distributions. For this channel type, the effect on open times may be larger than on type 1 channels. Thus, the effects of phosphorylation on channel gating are complex in that at least two open states and two closed states are modulated. Because these effects are very similar to those produced by altering $\mathrm{Ca}^{2+}$ concentrations, we examined the relationship between $\mathrm{Ca}^{2+}$ and phosphorylation. The open probability of a type 1 channel was examined as a function of the intracellular free $\mathrm{Ca}^{2+}$ concentration. The results are plotted in Figure 6 according to the Hill equation, showing a straight line (slope $=1.9$ ). Following reaction with $\mathrm{PK}-\mathrm{A}$, there is a shift in the $\mathrm{Ca}^{2+}$ dependence, the channel now being two to three times more sensitive to $\mathrm{Ca}^{2+}$

\begin{tabular}{|c|c|c|c|c|c|c|c|}
\hline & $n$ & $p_{o} \pm \mathrm{SE}$ & $\tau_{\mathrm{Cl}}{ }^{a}$ & $\tau_{\mathrm{C} 2}$ & $\tau_{\mathrm{C} 3}$ & $\tau_{01}$ & $\tau_{\mathrm{O} 2}$ \\
\hline \multicolumn{8}{|l|}{ Type 1 channels } \\
\hline Control & 6 & $0.44 \pm 0.102$ & $0.5(50)$ & $8.6(46)$ & $30.2(4)$ & $4.8(70)$ & $9.6(30)$ \\
\hline PK-A (400 nM) & 6 & $0.81 \pm 0.096$ & $0.5(71)$ & $4.6(26)$ & $11.5(3)$ & $6.7(68)$ & $11.2(32)$ \\
\hline Control & 5 & $0.55 \pm 0.121$ & $0.5(51)$ & $12.4(40)$ & $18.9(9)$ & $5.9(62)$ & $15.5(38)$ \\
\hline PP-2A (30 nM) & 5 & $0.19 \pm 0.086$ & $0.5(39)$ & $22.6(44)$ & $41.5(17)$ & $4.1(42)$ & $9.2(58)$ \\
\hline \multicolumn{8}{|l|}{ Type 2 channels } \\
\hline Control & 4 & $0.47 \pm 0.087$ & $0.5(71)$ & $67(16)$ & $140(13)$ & $23.2(48)$ & $40.6(30)$ \\
\hline PK-A (400 nM) & 4 & $0.07 \pm 0.067$ & $0.5(40)$ & $113(30)$ & $506(30)$ & $7.1(75)$ & $17.7(25)$ \\
\hline
\end{tabular}

Digitized single-channel records were analyzed by the $50 \%$ threshold-crossing method, and the individual closed times and open times from at least 5000 transitions were placed into 1 of 255 bins. The threshold value was calculated for each individual closed-to-open transition. The data were then fit by exponential functions, allowing the extraction of the time constants and percentages.

${ }^{a}$ Tau values are expressed in milliseconds, with the percentage shown in parentheses. 


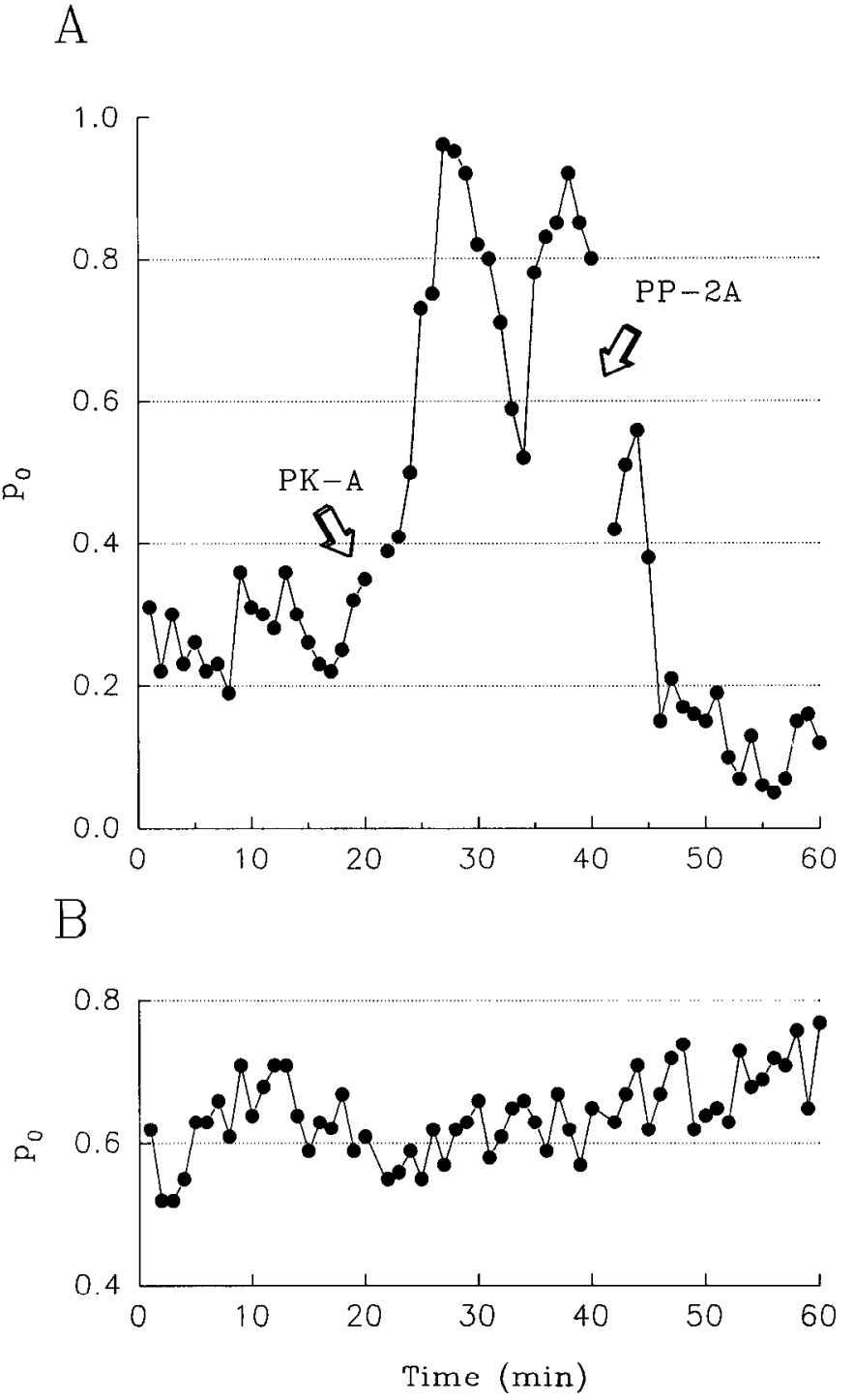

Figure 4. Time course of PK-A and PP-2A effects on the open probability of type $1 \mathrm{Ca}^{2+}$-activated $\mathrm{K}^{+}$channels. Single type 1 channels were incorporated into lipid bilayers as described in Figure 1. The open probability of these channels was adjusted to approximately 0.3 , using either $\mathrm{CaCl}_{2}$ or EGTA. Open probabilities were continuously calculated, and each point represents the value for a 60 -sec sweep. $A$, The arrows mark the time at which either $400 \mathrm{nM}$ PK-A or $30 \mathrm{~nm}$ PP-2A was added to the cytoplasmic side of the channel. $B$, The open probability of a channel was adjusted to approximately 0.5 , using either EGTA or $\mathrm{CaCl}_{2}$, and continuously monitored for $60 \mathrm{~min}$ without addition of any enzymes. Under such conditions, we never observed large sudden shifts in the open probability of single ion channels $(n=5)$.

(curve shifted to the left), apparently without a significant change in the number of $\mathrm{Ca}^{21}$ binding sites-(slope not changed). We were unable to examine the effect of phosphorylation on the $\mathrm{Ca}^{2+}$ dependence of type 2 channels, due to the experimental difficulties of obtaining single channels with minimal spontaneous open-probability fluctuations over a 1-2-hr period. In contrast to experiments with PK-A and PP-2A for which only end-point measurements are required, $\mathrm{Ca}^{2+}$ titration experiments require exceedingly stable recordings, which we were unable to obtain for this channel type.
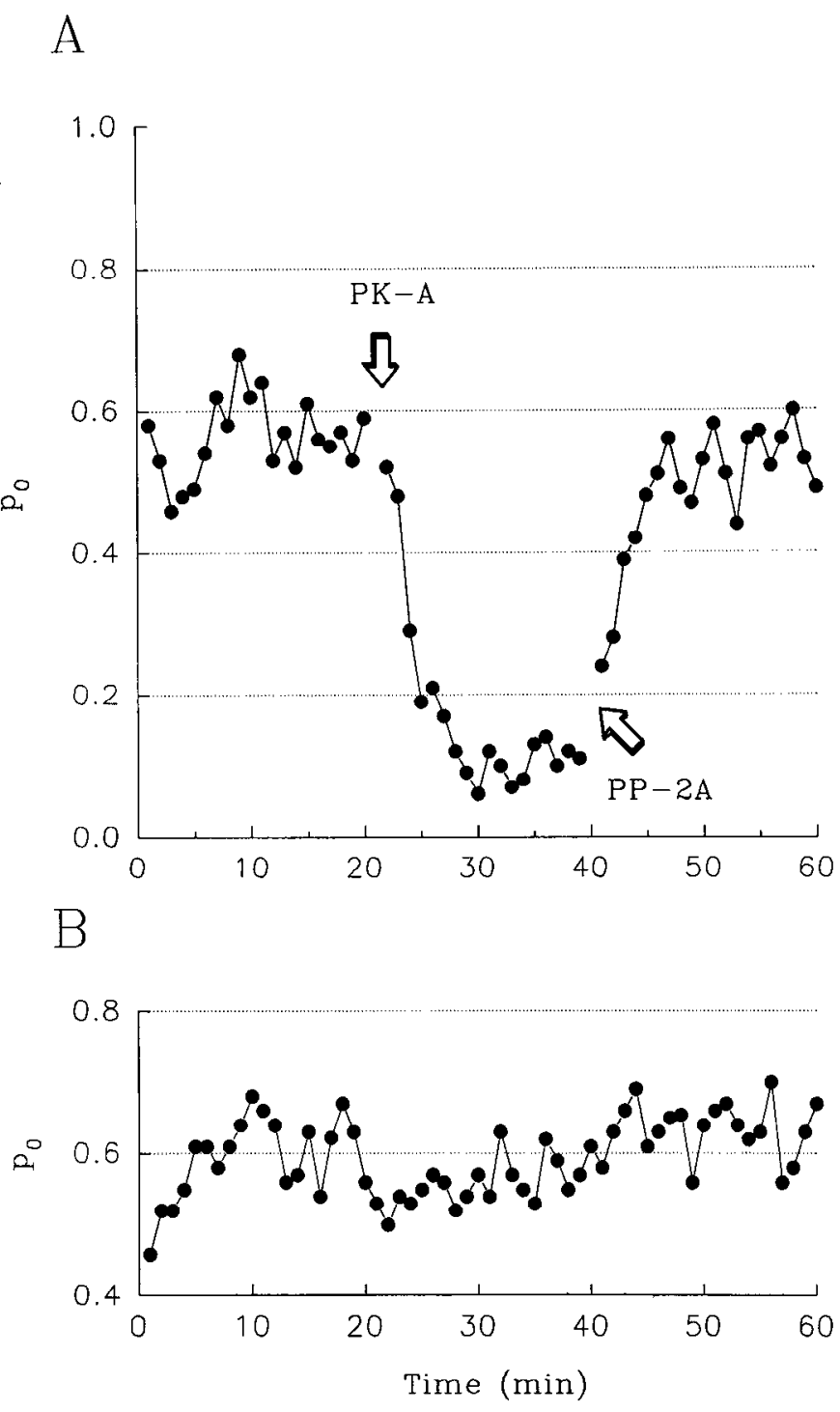

Figure 5. Time course of PK-A and PP-2A effects on the open probability of type $2 \mathrm{Ca}^{2+}$-activated $\mathrm{K}^{+}$channels. Single type 2 channels were incorporated into lipid bilayers as described in Figure 1. The open probability of these channels was adjusted to approximately 0.5 and measured as described in Figure 4. $A$, The arrows mark the time at which either $400 \mathrm{~nm}$ PK-A or $30 \mathrm{~nm}$ PP-2A was added to the cytoplasmic side of the channel. $B$, The open probability of a control channel was monitored for $60 \mathrm{~min}$ as described in Figure 4.

\section{Discussion}

Phosphorylation modulates maxi-K $K^{+}$channel gating

We have shown here that phosphorylation and dephosphorylation of maxi $\mathrm{Ca}^{2+}$-activated $\mathrm{K}^{+}$channels from rat brain causes upregulation and downregulation under defined conditions. Both type 1 and type 2 channel types can be modulated by a protein phosphorylation and dephosphorylation cycle, catalyzed by PK-A and PP-2A. The most consistent effect of PK-A on type 1 channels is to increase the open probability of these channels. The extent of this effect is dependent on the open probability of the channel prior to treatment. The largest effects are observed when initial open probabilities are between 0.3 and 0.6 . The effects of PK-A are minimized when the open probability is outside this range, as a result of changing either the holding 


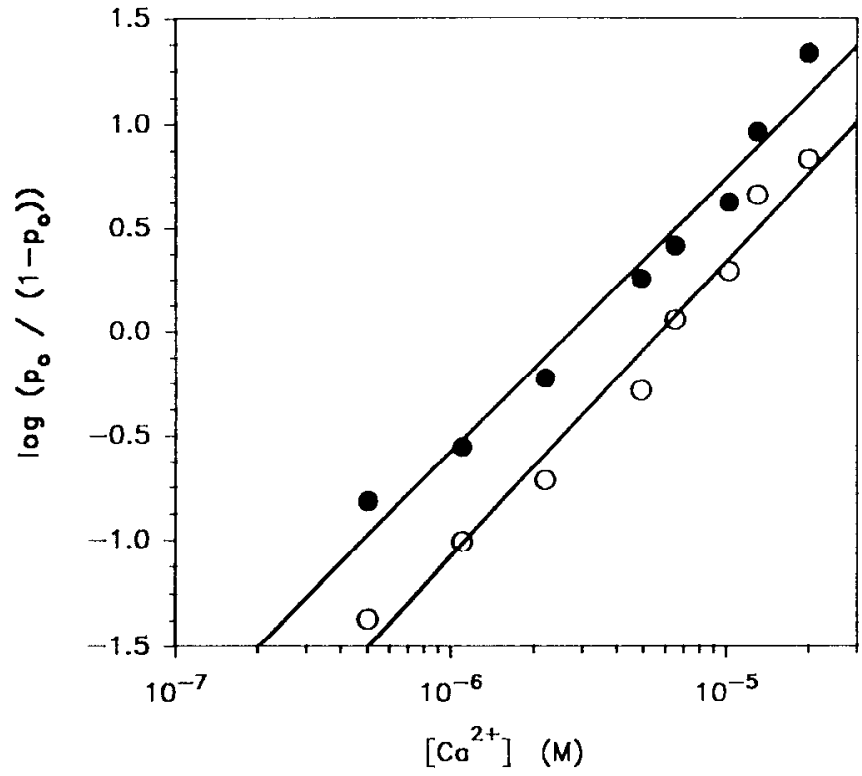

Figure 6. Phosphorylation increases the $\mathrm{Ca}^{2+}$ sensitivity of type 1 channels. Single type $1 \mathrm{Ca}^{2+}$-activated $\mathrm{K}^{+}$channels were incorporated into lipid bilayers as described in Figure 1 . The $\mathrm{Ca}^{2+}$ concentration on the cytoplasmic side of the channel was increased in a step-wise manner, and the open probability was calculated (open circles). The cytoplasmic side of the channel was then extensively perfused, 300 nM PK-A was added, and the $\mathrm{Ca}^{2+}$ concentration again changed in a step-wise manner (solid circles). The Hill coefficient for individual channels ranged between 1.4 and $2.5(n=5)$.

voltage or the free $\mathrm{Ca}^{2+}$ concentration. Upregulation of $\mathrm{Ca}^{2+}$ activated $\mathrm{K}^{+}$channels by $\mathrm{PK}-\mathrm{A}$ has been described in a number of tissues (Ewald et al., 1985; Farley and Rudy, 1988; Lechleiter et al., 1988; Sadoshima et al., 1988; Kume et al., 1989), and this may represent a common type of response to elevation of cellular cAMP levels. However, there is evidence that $\mathrm{Ca}^{2+}$ activated $\mathrm{K}^{+}$channels from pituitary gonadotrophs, like type 2 channels from brain, may also be downregulated by PK-A (Sikdar et al., 1989).

\section{Protein phosphatases can reverse the effects of $P K-A$}

An unexpected observation is the specificity of the purified catalytic subunit of PP-2A in reversing the effect of PK-A on both types of maxi-K ${ }^{+}$channels. Because PP- $2 \mathrm{~A}$ exactly reverses the effects of PK-A, the observed changes in gating kinetics must be due to protein phosphorylation. Significantly, PP-1 cannot mimic the cffects of PP-2A, though these phosphatases have been shown to have broad and overlapping specificities on a number of other substrate proteins (Ingebritsen and Cohen, 1983; Ingebritsen et al., 1983; Cohen, 1989). However, recent in vivo studies have demonstrated a similar degree of specificity for PP-1 versus PP-2A, where only PP-1 dephosphorylates myosin light chains to produce rearrangement of the microfilament network in fibroblasts (Fernandez et al., 1990). This specificity of phosphatases for maxi- $\mathrm{K}^{+}$channels opens the possibility that PP-2A is another locus for the modulation of ion channel activity. Evidence that there is partial endogenous phosphorylation of $\mathrm{Ca}^{2+}$ and $\mathrm{Na}^{+}$channels (Curtis and Catterall, 1985; Hosey et al., 1986; Armstrong and Eckert, 1987; Rossie and Catterall, 1987; De Jongh et al., 1989; Emerick and Agnew, 1989) and $\mathrm{Ca}^{2+}$-activated $\mathrm{K}^{+}$channels (present results) highlights the possible role of PP-2A in regulation. Regulation of phosphatase activity, even without a change in kinase activity, may lead to an alteration in channel-gating kinetics. It is known that brain contains significant amounts of all four major classes of phosphatases (PP-1, PP-2A, PP-2B, and PP-2C) bound to membranes (see Cohen, 1989), and that brain may also contain two novel phosphatases (LP-1 and LP-2), which have been proposed to play a unique role in the regulation of brain functions (Yang ct al., 1986, 1987).

\section{Phosphorylation alters the $\mathrm{Ca}^{2+} /$ voltage dependence of maxi- $K^{+}$channels}

To gain further insight into how phosphorylation alters channel gating, we have analyzed the effects of PK-A on channel opentime and closed-time distributions (Table 1). Channel gating could be described in terms of three closed states and two open states. As has been reported previously (McManus and Magleby, 1985), many channel closings are very brief ( $0.5 \mathrm{msec}$ or less) and are only poorly resolved in our experiments. The effect of PK-A on type 1 channels can be thought of as destabilizing at least one closed state and stabilizing at least one open state. The effects on the closed states are usually larger than the effects on the open states. This is similar to findings made in cultured smooth-muscle cells, where PK-A was also shown to change predominantly the closed state (Sadoshima et al., 1988). However, the latter experiments were carried out at very low open probabilities $\left(p_{o}=0.015-0.06\right)$, at which the channel open times were only approximately $1 \mathrm{msec}$, and small changes in the opentime distribution may not have been detected at a filter cutoff of $2 \mathrm{kHz}$.

Treating type 2 channels with PK-A has the opposite effect to that on type 1 channels: open states are destabilized, and closed states are stabilized. These alterations of both open-time and closed-time distributions (with little if any effect on the fast flickery closed state) are very similar to changes due to the addition of either $\mathrm{Ca}^{2+}$ or EGTA to channels (Magleby and Pallotta, 1983; Moczydlowski and Latorre, 1983; Vergara and Latorre, 1983; McManus and Magleby, 1985; Singer and Walsh, 1987). Hence, the kinetic "fingerprint" produced by phosphorylation is consistent with the possibility that phosphorylation alters the $\mathrm{Ca}^{2+}$ sensitivity of $\mathrm{Ca}^{2+}$-activated $\mathrm{K}^{+}$channels. This possibility, first proposed by De Peyer et al. (1982), was examined more directly by comparing the effect of PK-A on type 1 channels at a range of free $\mathrm{Ca}^{2+}$ concentrations. Phosphorylation seems to increase the $\mathrm{Ca}^{2+}$ affinity of the channel by a factor of two to three; that is, less $\mathrm{Ca}^{2+}$ is required to open the phosphorylated channel to any given open probability. The number of $\mathrm{Ca}^{2+}$ ions involved in channel upregulation does not appear to change after PK-A treatment, as judged by the similarity in Hill coefficients. Thus, phosphorylation of this type of channel may not alter channel gating per se, but rather alter gating indirectly by stabilizing the binding of $\mathrm{Ca}^{2+}$. Alternatively, our data could be interpreted to indicate that the voltage dependence of the channels is being modulated. The voltage dependence of these channels is not clearly understood, and it is possible that the $\mathrm{Ca}^{2}$-binding step itself may be voltage sensitive (Moczydlowski and Latorre, 1983; but see Methfessel and Boheim, 1982, for an alternative proposal). Hence, phosphorylating a "voltage-sensor" region of the channel could induce a significant alteration in the voltage and calcium dependence.

A correlation between phosphorylation and the shift in the $\mathrm{Ca}^{2+}$ sensitivity of maxi-K $\mathrm{K}^{+}$channels may account for the large differences in the $\mathrm{Ca}^{2+}$ sensitivities of these channels observed 
by numerous workers (Marty, 1983, 1989; Singer and Walsh, 1986; Wolff et al., 1986). Biochemical studies on the phosphorylation of $\mathrm{Ca}^{2+}$ and $\mathrm{Na}^{+}$channels have revealed that PK-A often produces little or no incorporation of phosphate into channel subunits because the target residues are already phosphorylated when the channel is isolated (Rossie and Catterall, 1987; Emerick and Agnew, 1989).

\section{Modulation may involve multiple phosphorylation sites}

The time delay between the addition of PK-A and the onset of any change in gating kinetics is clearly longer than for $\mathrm{Ca}^{2+}$ or EGTA, indicating either that PK-A must overcome some diffusion barrier, that multiple sites must be phosphorylated before gating is affected, or that an endogenous phosphatase activity is competing with the PK-A activity. We believe that, because a shift in gating can take some minutes before a maximal effect is observed, and because the shift is gradual rather than occurring in a discrete jump, multiple sites are being phosphorylated in individual ion channels. For $\mathrm{Na}^{+}$channels, $\mathrm{Ca}^{2+}$ channels, and $\mathrm{ACh}$ receptor/channels, it is known that multiple phosphorylation sites exist (Huganir et al., 1986; Rossie et al., 1987; Catterall et al., 1988; Hosey and Lazdunski, 1988; De Jongh et al., 1989; Rossie and Catterall, 1989).

To examine whether the channel itself is being phosphorylated, rather than some intermediate "regulator" protein which in turn modulates the channel, we extensively perfused the cytoplasmic side of the channel after vesicle fusion, thereby removing any unfused vesicles or soluble regulator proteins. These conditions did not significantly alter the actions of PK-A on either type 1 or type 2 channels. Because any proteins incorporated into the planar bilayer are essentially at infinite dilution, this indicates that phosphorylation of either the channel itself or a tightly associated protein is responsible for channel modulation.

After PK-A has induced a shift in gating kinetics, we often see large fluctuations in the open probability of individual ion channels. These fluctuations are on a time scale of many seconds to minutes and may indicate either that the effects of PK-A on channel gating are very complex, allowing the channel to move between a number of kinetic states, or that a phosphatase activity tightly associated with the channel is bringing the open probability back to control levels. This latter possibility suggests that the phosphorylated and dephosphorylated states of an ion channel are actively "cycled," so small changes in the activity of either the kinase or the phosphatase can result in rapid changes in the phosphorylation state, and hence the activity of the channel. Evidence that such mechanisms may occur in vivo has recently been presented by Klumpp et al. (1990). These workers made use of okadaic acid, a membrane-permeable polyether inhibitor of PP-1 and PP-2A, to show that PP-1 is responsible for dephosphorylation and closure of calcium channels in Paramecium. It has been proposed that such dephosphorylated channels represent a "closed-inactivated" state and must be rephosphorylated to become resensitized to depolarizing stimuli (Armstrong and Eckert, 1987; Klumpp et al., 1990). In another study on tracheal myocytes, okadaic acid was shown to activate $\mathrm{Ca}^{2+}$-activated $\mathrm{K}^{+}$channels in cell-attached membrane patches (Kume et al., 1989). These findings indicate that in some cell types an ion channel phosphorylation/dephosphorylation cycle is continuously active, and that channel gating can be altered by modulating either the kinase or the phosphatase portion of the cycle.

\section{Families of modulatable maxi- $K^{+}$channels}

The properties of large-conductance $\mathrm{Ca}^{2+}$-activated $\mathrm{K}^{+}$channels from a wide variety of tissues appear to be very similar (Latorre and Miller, 1983; Latorre, 1986; Singer and Walsh, 1986; Wolff et al., 1986; Marty, 1989). However, our previous characterization of four types of $\mathrm{Ca}^{2+}$-activated $\mathrm{K}^{+}$channels from rat brain revealed the existence of at least two types of maxi- $\mathrm{K}^{+}$ channels differing in their gating kinetics, $\mathrm{Ca}^{2+}$ sensitivity, and sensitivity to CTX (Reinhart et al., 1989). These differences between maxi- $\mathrm{K}^{+}$channels are further highlighted in the present study showing that PK-A can upregulate one and downregulate the other maxi-K $\mathrm{K}^{+}$channel type from brain. Hence, there may be a family of modulatable maxi- $\mathrm{K}^{+}$channels in brain, differing not only in toxin-binding and gating properties, but also in their response to phosphorylation. Even channels that appear kinetically and pharmacologically similar may be affected in very different ways by protein phosphorylation. Similarly, channels that have common toxin binding sites may be kinetically distinct (MacKinnon et al., 1988).

This concept of families of closely related potassium channels is highlighted by the large number of channel types isolated from rat brain cDNA libraries by cross-hybridization with a Shaker cDNA probe (Baumann et al., 1988; Iverson et al., 1988; Tempel et al., 1988; Stuehmer et al., 1989; Koren et al., 1990). It was surprising to find that even very minor structural variations have been found to underlie differences in gating properties and the sensitivity to some blocking agents (Iverson et al., 1988; Kamb et al., 1988; Timpe et al., 1988; Pongs, 1989). Similarly, the cloning of $\mathrm{Na}^{+}$channels has revealed a family of different $\alpha$-subunits (Noda et al., 1986). Subunits of neuronal origin were found to be unique in that they contain an insert embracing a cluster of PK-A phosphorylation sites (Rossie et al., 1987; Rossie and Catterall, 1989). The phosphorylation of these channels has been implicated in an altered binding to neurotoxins and may modulate the voltage dependence of inactivation (Rossie and Catterall, 1987). Furthermore, two different forms of L-type $\mathrm{Ca}^{2+}$-channel $\alpha$-subunits have recently been characterized (De Jongh et al., 1989), only one of which contains a C-terminal segment embracing multiple potential PK-A phosphorylation sites. Hence, while the ion-conduction and voltage-sensing components of many ion channels may be similar, there may be considerable variation in modulatory components of ion channels.

Finally, the results presented in this report highlight how diverse responses to a single second-messenger system can be gencrated. Different cell types, or regions within a cell, could respond to PK-A by either upregulating or downregulating maxi$\mathrm{K}^{+}$channels in response to a given stimulus. The magnitude and duration of the effect can be precisely modulated by setting the phosphorylation state of multiple sites in a channel not only by modulating the kinase activity, but also by modulating the phosphatase activity. If multiple phosphorylation sites modulate channel gating, then one can envision schemes in which not only the total number of phosphate groups, but also the order of phosphorylation and dephosphorylation that would generate different combinations of phosphate groups, may take on a special significance. The possibility of functional complexes involving ion channels, together with kinase and phosphatase activities, allows for an even greater diversity of responses to extracellular stimuli than that already afforded by the great diversity of different $\mathrm{K}^{+}$channel types. 


\section{References}

Armstrong D, Eckert R (1987) Voltage-activated calcium channels that must be phosphorylated to respond to membrane depolarization. Proc Natl Acad Sci USA 84:2518-2522.

Baraban JM, Snyder SH, Alger BE (1985) Protein kinase C regulates ionic conductance in hippocampal pyramidal neurons: electrophysiological effects of phorbol esters. Proc Natl Acad Sci USA 82:25382542.

Baumann A, Grupe A, Achermann A, Pongs O (1988) Structure of the voltage dependent potassium channel is highly conserved from Drosophila to vertebrate central nervous system. EMBO J 7:24572463.

Blatz AL, Magleby KL (1986) Single apamin-blocked Ca ${ }^{2+}$-activated $\mathrm{K}^{+}$channels of small conductance in cultured rat skeletal muscle. Nature 323:718-720.

Brautigan DL, Shriner CL, Grippuso PA (1985) Phosphorylase phosphatase catalytic subunit. Evidence that the $M_{r}=33,000$ enzymatic fragment is derived from a native protein of $M_{r}=70,000$. J Biol Chem 260:4295-4302.

Castle NA, Haylett DG, Jenkinson DH (1989) Toxins in the characterization of potassium channels. Trends Neurosci 12:59-65.

Catterall WA, Seagar MJ, Takahashi M (1988) Molecular properties of dihydropyridine-sensitive calcium channels in skeletal muscle. $\mathrm{J}$ Biol Chem 263:3535-3538.

Cohen P (1989) The structure and regulation of protein phosphatases. Annu Rev Biochem 58:453-508.

Curtis BM, Cattcrall WA (1985) Phosphorylation of the calcium antagonist receptor of the voltage-sensitive calcium channel by cAMPdependent protein kinase. Proc Natl Acad Sci USA 82:2528-2532.

De Jongh KS, Merrick DK, Catterall WA (1989) Subunits of purified calcium channels: a 212-kDa form of $\alpha_{1}$ and partial amino acid sequence of a phosphorylation site of an independent $\beta$ subunit. Proc Natl Acad Sci USA 86:8585-8589.

Demaille J, Peters KA, Fisher EH (1977) Isolation and properties of rabbit skeletal muscle protein inhibitor of adenosine $3^{\prime}, 5^{\prime}$-monophosphate dependent protein kinases. Biochemistry 16:3080-3086.

De Peyer JE, Cachelin AB, Levitan IB, Reuter H (1982) $\mathrm{Ca}^{2+}$-activated $\mathrm{K}^{+}$conductance in internally perfused snail neurons is enhanced by protein phosphorylation. Proc Natl Acad Sci USA 79:4207-421 1.

Doerner D, Pitler TA, Alger BA (1988) Protein kinase C activators block specific calcium and potassium current components in isolated hippocampal neurons. J Neurosci 8:4069-4078.

Elkins T, Ganetzki B, Wu C-F (1986) A Drosophila mutation that eliminates a calcium-dependent potassium current. Proc Natl Acad Sci USA 83:8415-8419.

Emerick MC, Agnew WS (1989) Identification of phosphorylation sites for adenosine $3^{\prime}, 5^{\prime}$-cyclic phosphate dependent protein kinase on the voltage-sensitive sodium channel from Electrophorus electricus. Biochemistry 28:8367-8380.

Ewald D, Williams A, Levitan IB (1985) Modulation of single $\mathrm{Ca}^{2+}$ dependent $\mathrm{K}^{+}$channel activity by protein phosphorylation. Nature 315:503-506.

Farley J, Rudy B (1988) Multiple types of voltage-dependent $\mathrm{Ca}^{2+}$ activated $\mathrm{K}^{+}$channels of large conductance in rat brain synaptosomal membranes. Biophys J 53:919-934.

Fernandez A, Brautigan DL, Mumby M, Lamb NJC (1990) Protein phosphatase type-1, not type-2A, modulates actin microfilament integrity and myosin light chain phosphorylation in living nonmuscle cells. J Cell Biol 111:103-112.

Hille B (1984) Ionic channels of excitable membranes. Sunderland, MA: Sinauer.

Hosey MM, Borsotto M, Lazdunski M (1986) Phosphorylation, and dephosphorylation of dihydropyridine-sensitive voltage-dependent $\mathrm{Ca}^{2+}$ channel in skeletal muscle membranes by cAMP- and $\mathrm{Ca}^{2+}$ dependent processes. Proc Natl Acad Sci USA 83:3733-3737.

Hosey MM, Lazdunski M (1988) Calcium channels: molecular pharmacology, structure and regulation. J Membr Biol 104:81-105.

Huganir RL, Delcour AH, Greengard P, Hess GP (1986) Regulation of desensitization of the nicotinic acetylcholine receptor by protein phosphorylation. Nature 321:774-776.

Ingebritsen TS, Cohen P (1983) The protein phosphatases involved in cellular regulation. 1. Classification and substrate specificities. Eur $\mathrm{J}$ Biochem 132:255-261.
Ingebritsen TS, Stewart AA, Cohen P (1983) The protein phosphatases involved in cellular regulation. 6. Measurement of type- 1 and type- 2 protein phosphatases in extracts of mammalian tissues; an assessment of their physiological roles. Eur J Biochem 132:297-307.

Iverson LE, Tanouye MA, Lester HA, Davidson N, Rudy B (1988) Potassium channels from Shaker RNA expressed in Xenopus oocytes. Proc Natl Acad Sci USA 85:5723-5727.

Kaczmarek LK (1988) The regulation of neuronal calcium and potassium channels by protein phosphorylation. Adv Sec Mess Phosp Res 22:113-139.

Kamb A, Tseng-Crank J, Tanouye MA (1988) Multiple products of the Drosophila Shaker gene may contribute to potassium channel diversity. Neuron 1:421-430.

Klumpp S, Cohen P, Schultz JE (1990) Okadaic acid, an inhibitor of protein phosphatase 1 in Paramecium, causes sustained $\mathrm{Ca}^{2+}$-dependent backward swimming in response to depolarizing stimuli. EMBO J 9:685-689.

Koren G, Liman ER, Logothetis DE, Nadal-Ginard B, Hess P (1990) Gating mechanism of a cloned potassium channel expressed in frog oocytes and mammalian cells. Neuron $2: 39-51$.

Kume H, Tokuno H, Tomita T (1989) Regulation of $\mathrm{Ca}^{2+}$-dependent $\mathrm{K}^{+}$-channels in tracheal myocytes by phosphorylation. Nature 341 : $152-154$.

Latorre R (1986) The large calcium-activated potassium channel. In: Ion channel reconstitution (Miller C, ed), pp 431-467. New York: Plenum.

Latorre R, Miller C (1983) Conduction and selectivity in potassium channels. J Membr Biol 71:11-30.

Lechleiter JD, Dartt DA, Brchm P (1988) Vasoactive intestinal peptide activates $\mathrm{Ca}^{2+}$-dependent $\mathrm{K}^{+}$channels through a cAMP pathway in mouse lacrimal cells. Neuron 1:227-235.

MacKinnon R, Reinhart PH, White MM (1988) Charybdotoxin block of Shaker $\mathrm{K}^{+}$channels expressed in Xenopus oocytes suggests that functionally different $\mathrm{K}^{+}$channels share common structural features. Neuron 1:997-1001.

Magleby KL, Pallotta BS (1983) Calcium dependence of open and shut interval distributions from calcium-activated potassium channels in cultured rat muscle. J Physiol (Lond) 344:585-604.

Marty A (1983) $\mathrm{Ca}^{2+}$-dependent $\mathrm{K}^{+}$-channels with large unitary conductance. Trends Neurosci 6:262-265.

Marty A (1989) The physiological role of calcium-dependent channels. Trends Neurosci 12:420-424.

McManus OB, Magleby KL (1985) The large-conductance calciumactivated potassium channel in cultured rat muscle has at least three open states of similar conductance and six shut states. Biophys J 47 : $137 a$.

Methfessel C, Boheim G (1982) The gating of single calcium-dependent potassium channels is described by an activation/blockade mechanism. Biophys Struct Mech 9:35-60.

Moczydlowski E, Latorre R (1983) Gating kinetics of $\mathrm{Ca}^{2+}$-activated $\mathrm{K}^{+}$channels from rat muscle incorporated into planar lipid bilayers. J Gen Physiol 82:511-542.

Moczydlowski E, Lucchesi K, Ravindran A (1988) An emerging pharmacology of peptide toxins targeted against potassium channels. J Membr Biol 105:91-111.

Mueller P, Rudin DO (1969) Translocators in bimolecular lipid membranes: their role in dissipative and conservative energy transduction. Curr Top Bioenerg 3:157-249.

Noda M, Ikeda T, Kayono T, Suzuki H, Takeshima T, Kurasaki M, Takahashi H, Numa S (1986) Existence of distinct sodium channel messenger RNAs in rat brain. Nature 320:188-192.

Pallis DC, Shahrik LK, Martin BL, Jaspers S, Miller TB, Brautigan DL, Roberts TM (1990) Polyoma small and middle T antigens and SV40 small $T$ antigen form stable complexes with protein phosphatase 2A. Cell 60:167-176.

Pennefather P, Lancaster B, Adams PR, Nicoll RA (1985) Two distinct $\mathrm{Ca}$-dependent $\mathrm{K}$ currents in bullfrog sympathetic ganglion cells. Proc Natl Acad Sci USA 82:3040-3044.

Peters K, Demaille J, Fisher E (1977) Adenosine 3':5'-monophosphate dependent protein kinase from bovine heart. Characterization of the catalytic subunit. Biochemistry 16:5691-5697.

Petersen OH, Maruyama Y (1984) Calcium-activated potassium channels and their role in secretion. Nature 307:693-696.

Pongs O (1989) Molecular basis of potassium channel diversity. Pfluegers Arch 414:S71-S75. 
Reinhart PH, Levitan IB (1987) Modulation of charybdotoxin-sensitive calcium-dependent potassium channels by phosphorylation. Soc Neurosci Abstr 13:1439.

Reinhart PH, Chung S, Levitan IB (1989) A family of calcium-dependent potassium channels from rat brain. Neuron 2:1031-1041.

Romey G, Lazdunski M (1984) The cocxistence in rat muscle cells of two distinct classes of $\mathrm{Ca}^{2+}$-dependent $\mathrm{K}^{+}$-channels with different pharmacological properties and different physiological functions. Biochem Biophys Res Commun 118:669-674.

Rossie S, Catterall WA (1987) Cyclic AMP-dependent phosphorylation of voltage-sensitive sodium channels in primary cultures of rat brain neurons. J Biol Chem 262:12735-12744.

Rossie S, Gordon D, Catterall WA (1987) Identification of an intracellular domain of the sodium channel having multiple cAMP-dependent phosphorylation sites. J Biol Chem 262:17530-17535.

Rossie S, Catterall WA (1989) Phosphorylation of the $\alpha$ subunit of rat brain sodium channels by cAMP-dependent protein kinase at a new site containing Ser ${ }^{686}$ and Ser $^{687}$. J Biol Chem 264:14220-14224.

Rudy B (1988) Diversity and ubiquity of K channels. Neuroscience 25:729-749.

Sadoshima J, Akaike N, Kanaide H, Nakamura M (1988) Cyclic AMP modulates Ca-activated $\mathrm{K}$ channel in cultured smooth muscle cells of rat aortas. Am J Physiol 255:H754-H759.

Shearman MS, Sekiguchi K, Nishizuka Y (1989) Modulation of ion channel activity: a key function of the protein kinase Cenzyme family. Pharmacol Rev 41:211-237.

Sikdar SK, McIntosh RP, Mason WT (1989) Differential modulation of $\mathrm{Ca}^{2+}$-activated $\mathrm{K}^{+}$-channels in ovine pituitary gonadotrophs by GnRH, $\mathrm{Ca}^{2+}$ and cyclic AMP. Brain Res 496:113-123.
Singer JJ, Walsh JV (1986) Large-conductance $\mathrm{Ca}^{2+}$-activated $\mathrm{K}^{+}$ channels in freshly dissociated smooth muscle cells. Membr Biochem 6:83-110.

Singer JJ, Walsh JV (1987) Characterization of calcium-activated potassium channels in single smooth muscle cells using the patch-clamp technique. Pfluegers Arch 408:98-111.

Stuehmer W, Ruppersberg JP, Schroeter KH, Sakmann B, Stocker M, Giese KP, Perschke A, Baumann A, Pongs O (1989) Molecular basis of functional diversity of voltage-gated potassium channels in mammalian brain. EMBO J 8:3235-3244.

Tempel BL, Yan YN, Jan LY (1988) Cloning of a probable potassium channel gene from mouse brain. Nature 332:837-839.

Timpe LC, Schwarz TL, Tempel BL, Papazian DM, Jan YN, Jan LY (1988) Expression of functional potassium channels from Shaker cDNA in Xenopus oocytes. Nature 331:143-145.

Vergara C, Latorre $\mathbf{R}$ (1983) Kinetics of $\mathrm{Ca}^{2+}$-activated $\mathrm{K}^{+}$channels from rabbit muscle incorporated into planar bilayers. J Gen Physiol 82:543-568

Wolff D, Vergara C, Cecchi X, Latorre R (1986) Characterization of large-unitary-conductance calcium-activated potassium channels in planar lipid bilayers. In: Ionic channels in cells and model systems (Latorre R, ed), pp 307-334. New York: Plenum.

Yang S-D, Yu J-S, Fong Y-L (1986) Purification and characterization of two inactive/latent protein phosphatases from pig brain. J Biol Chem 261:5590-5596.

Yang S-D, Liu J-S, Fong Y-L, Yu J-S, Tzen T-C (1987) Endogenous basic protein phosphatases in the brain myelin. J Neurochem 48:160166. 